\title{
An Analysis of Induced Pressure Fields in Electroosmotic
}

\section{Flows through Microchannels}

\author{
Yonghao Zhang*, Xiao-Jun Gu, Robert W. Barber and David R. Emerson \\ The Center for Microfluidics, The Central Laboratory of Research Councils, Daresbury \\ Laboratory, Warrington, WA4 4AD, UK
}

\begin{abstract}
Induced pressure gradients are found to cause band-broadening effects which are important to the performance of microfluidic devices such as capillary electrophoresis and capillary chromatography. An improved understanding of the underlying mechanisms involved in causing an induced pressure gradient in electroosmotic flows is presented. The analysis shows that the induced pressure distribution is the key to understanding the experimentally observed phenomena of leakage flows. A novel way of determining the static pressures at the inlet and outlet of microchannels is also presented that takes account of the pressure losses due to flow contraction and expansion. These commonly neglected pressure losses at the channel entrance and outlet are shown to be important in accurately describing the flow. The important parameters that define the effect of an induced pressure on the flows are discussed, which may facilitate the design of improved microfluidic devices. The present model clearly identifies the mechanism behind the experimentally observed leakage flows, which is further confirmed by numerical simulations. Not only can the leakage flow occur from the electric-field free side channel to main channel, but also the fluid in the main channel can be attracted into the side channel by the induced pressure gradient.
\end{abstract}

\footnotetext{
*Tel: 44-1925-603149; Fax: 44-1925-603634; Email: Y.Zhang@dl.ac.uk
} 
Key words: electroosmotic flow; microchannel; boundary conditions; creeping flow; entry flow.

\section{INTRODUCTION}

Experimental techniques associated with electroosmotic flow (EOF) through micro-channels have been widely applied, e.g. capillary electrophoresis [1-5] and capillary chromatography [69]. The benefits of these microfluidic devices are the dramatic reduction of reagent consumption, analysis time and sample dispersive effects. In an ideal EOF, the velocity profile is plug-like and the mean velocity is independent of the cross-sectional area if the electric double-layer is much smaller than the characteristic length scale of the channel. In a conventional pressure-driven flow, the velocity profile is parabolic and the mean velocity depends on the cross-sectional area of the channel. Therefore, an EOF offers significantly less deleterious dispersive effects than a pressure-driven flow in a channel. With appropriate application of electric potentials, its valveless control of fluid flow is a favored highperformance sample separation technique [10-13].

However, an EOF is difficult to control due to complex nature of surface composition, buffer characteristics and external electric fields [14]. Apart from these factors, the control of the EOF is also affected by fluid hydrodynamics. One problem that arises when using an EOF in channels with intersections is the contamination of the sample by the stationary liquid in the side-channels. Leakage flows have been observed experimentally $[1,2,10,15,16]$ from electrical-field free side channels into the main streams where electric fields were applied. In these observations, the amount of leakage depended on the layout of the channels and it was attributed to hydrodynamic effects other than molecular diffusion. Because the side channel is 
electric-field free, the leakage flow must be driven by a pressure gradient. Understanding the pressure distribution generated along the primary flow field is therefore the key to explaining this experimentally observed phenomenon. However, measuring the pressure field at such small scales, without disturbing the flow field, is very challenging. Theoretical predictions and numerical simulations can therefore play an important role in tackling this issue and recent theoretical and numerical analysis of EOFs in channels [17-27] has been making headway.

Previous simulations [20, 23] of a steady fluid flow in both straight and cross-sectional channels have assumed that the pressures at the entrance and the outlet were at atmospheric conditions. In a straight channel as shown in Figure 1a, the pressure gradient along the flow direction is therefore assumed zero by many researchers [19, 20, 23, 28]. However, as pressure can be induced by the flow, the pressure gradient along the channel needs to be considered. The physical mechanism is that the viscous dissipation of the fluid will cause an irreversible pressure loss at the channel inlet and outlet where flow contraction and expansion occur, which leads to a pressure difference at the channel inlet and outlet despite there being no applied pressure in the reservoirs. Because the fluid is driven out of the channel by a pressure gradient, the "electroosmotic pump" needs to build up this amount of pressure in the channel. The schematic diagram of pressure distribution along a straight channel can be seen in Figure $1 \mathrm{~b}$. The induced pressure gradient is dependent on the pressure losses, which needs to be determined as boundary conditions in the simulation. As a result, the assumption of environmental pressure at both channel inlet and outlet may lead to significant error and underestimate dispersive effects. Furthermore, the simulations with such pressure boundary conditions for intersectional channels failed to predict the leakage flow from the side channel that had been observed experimentally. A more recent simulation [25] adopted a different set 
of boundary conditions, where a zero pressure gradient was assumed at the channel inlet. This is physically insufficient because the fluid is drawn into the channel by a pressure gradient.

These two typical types of boundary conditions used in both Patankar and $\mathrm{Hu}$ [20] and Yang et al. [25] decoupled the flow at the channel entrance from the oncoming flow from the far field of the reservoirs. The error caused by these assumptions is not known [29], and the detected pressure rise by Yang et al. [25] may be attributed to the inaccuracy of the assumptions of boundary conditions. An understanding of the flow mechanism is therefore essential to establish an accurate and more physically representative numerical model.

Our analysis leads us to believe that the observed leakage is due to the pressure distribution which is caused by the fluid motion initially from rest. In this paper, the analysis focuses on pressure losses at the channel inlet and outlet which helps us to establish the boundary conditions. As these boundary conditions are applied to the flow in a straight channel, the key parameters that affect the flow dispersion will be clearly identified. Afterwards, an EOF through a T-shaped channel that has no externally applied pressure in the reservoirs is numerically analyzed where the key factors associated with the leakage flow in general will be discussed.

In order to exclude other factors which affect the control of an EOF, we assume the zeta potential at the channel surface is ideally uniform and the electric double layer is negligibly smaller than the characteristic length scale of the channel. The fluid is assumed Newtonian and the flow is laminar. 


\section{MATHEMATICAL MODEL}

The Navier-Stokes equations can be employed to describe EOFs with a body force acting on the ions by the electrical field. The governing equations for an EOF are given by:

continuity equation:

$$
\nabla \cdot \boldsymbol{U}=0
$$

momentum equation:

$$
\rho \frac{D \boldsymbol{U}}{D t}=-\nabla p+\mu \nabla^{2} \boldsymbol{U}+\boldsymbol{F},
$$

where $\boldsymbol{U}$ is the velocity; $\rho$, the fluid density; $p$, the static pressure; $\mu$, the viscosity. $\boldsymbol{F}$ is the electrical force acting on fluid, which is given by [20]

$$
\boldsymbol{F}=-\rho_{e} \nabla(\psi+\phi),
$$

where $\rho_{e}$ is the electric charge density; $\psi$, the electric potential due to the zeta potential at the wall; $\phi$, the applied electric field.

The applied electric field can be described by the Laplace equation, i.e. [20]

$$
\nabla^{2} \phi=0
$$

The local net charge density $\rho_{e}$ can be given by [20]

$$
\nabla^{2} \psi=-\rho_{e} / \varepsilon,
$$

where $\varepsilon$ is the electric permittivity of the solution. The classical Poisson-Boltzmann equation is used to govern the distribution of the electric potential, $\psi$, as

$$
\nabla^{2} \psi=k^{2} \psi,
$$

where $k^{-1}$ is called the Debye length, which is used to describe the characteristic thickness of the double layer. Substituting Eq. [6] into Eq. [5], we get

$$
\rho_{e}=-\varepsilon k^{2} \psi
$$


The details and limitation of the adopted theory for EOFs can be referred to Hunter [30].

\section{PRESSURE LOSSES AT CHANNEL INLET AND OUTLET}

In order to determine the flow field of an EOF, we need to solve equations [1-7] with proper boundary conditions at the channel inlet and outlet. Eq. [4] is independently solved for the applied electric field, $\phi$. With $\rho_{e}$ and $\psi$ determined by Eqs $[6,7]$, the electric force acting on the fluid is readily obtained by solving Eq. [3]. Afterwards, a standard Navier-Stokes flow solver can be used to solve equations $[1,2]$ for the fluid velocity and pressure fields. However, the boundary conditions at the channel inlet and outlet have been an arguable issue for the simulation of microfluidic flows. For example, for a steady flow without imposed pressure on the reservoirs, Patankar and $\mathrm{Hu}[20]$ asserted the atmospheric pressure at the channel inlet and

outlet. Recently, Yang et al. [25] assumed a zero pressure gradient in the channel longitudinal direction at the inlet. These boundary conditions effectively decoupled the flow at the entrance from the oncoming flow outside the channel. The error caused by these assumptions might be small for a pressure driven flow especially with high Reynolds number. However, for creeping flows through microchannels driven by an electrical field, the error may lead to significant inaccuracy in predicting flow behavior. Due to the importance of the pressure field to a microfluidic flow, the pressure drop from the far field to the entrance must be taken into account as well as the amount of pressure drop needed to drive the flow out of the channel to the reservoirs.

If we solve Eqs [1-7] for a straight channel with two connected reservoirs (as shown in figure 1), typical pressure contours at the channel inlet and outlet are given by figure 2 (for illustration purpose only). Here, we have used our own flow solver- $\mu$-Thor 2.0 and the flow 
solver of CFD-ACE [31], which employ classical SIMPLE algorithms. Both solvers give the same answers for the creeping flows considered in this paper. The details of the SIMPLE algorithms are widely available, e.g. Ferziger and Perić [32]. Because the atmospheric pressure conditions can be applied at the free surface of the reservoirs, the error introduced by asserting boundary conditions at the channel inlet and outlet is avoided. However, computational cost is huge because the size of reservoirs is usually orders larger than the microchannel depth. We can see that there are flow development regimes at the channel inlet and outlet where the pressure distribution is not uniform in the $y$ direction. However, the flow development length, $l_{d}$, is small in comparison to the channel length, $L$, for creeping flows. For a pressure driven creeping flow, the flow development length, $l_{d}$, is $0.63 d$, where $d$ is the thickness of channel [33]. Therefore, fully-developed flow status is quickly established and the pressure becomes uniform in the $y$ direction (outside of the electric double layer). If we can determine the pressure at the end of the flow development regime, the pressure boundary conditions at the channel inlet can be established where the pressure distribution in the $y$ direction is uniform (see figure 2). This approach effectively sidesteps the problematical flow development regime but still captures the channel end effect on the channel main flow regime. If the prime interest is within the flow development regimes at the channel inlet and outlet, this approach is not appropriate. Otherwise, the pressure at the inlet can be determined as

$$
p_{\text {in }}=P_{0}-\Delta P_{\text {in }},
$$

where $p_{i n}$ is the pressure at the end of flow development regime so that it is uniform in the $y$ direction, and

$$
\Delta P_{i n}=\rho \frac{1}{2} \bar{u}_{i n}^{2}+\delta p_{i n}
$$

where $\bar{u}_{i n}$ and $\delta p_{i n}$ are the mean velocity and the pressure loss at the entrance respectively. The first term on the right hand is the reversible kinetic energy which can be neglected for a 
creeping flow. The expression for the pressure loss $\delta p_{i n}$ depends on the channel geometry. For a creeping flow through a infinitely thin slit, the total pressure loss due to flow contraction and expansion has been determined theroetically by Roscoe [34]

$$
\delta p_{\text {total }}=\frac{32}{\pi} \frac{\mu \bar{U}}{d}
$$

where $\bar{U}$ is the mean velocity in the slit. Again, the inertial effect on the pressure loss is negligible. The pressure losses due to flow contraction and expansion through a slit are very similar to those at the channel inlet and outlet, so that Eq. [10] can be used to determine the pressure boundary conditions for a channel.

Because we consider a flow through a long channel with small flow development length here (the boundary conditions at the inlet are to be established at the end of small flow development regime), apart from the pressure loss caused by flow contaction, the extra pressure loss due to friction with the channel wall and the extra pressure gain due to the work done by the electric force on fluid have to be taken into account. However, since the flow development length is small ( $l_{d}=0.63 d$ for a pressure driven creeping flow), the pressure loss, $\delta p_{f}$, due to friction with the channel wall in this regime may be less significant than the pressure loss due to the flow contraction. Moreover, once the fluid enters the channel, the work done by the electric force on the fluid will build up a pressure, $\delta p_{e}$, which may offset the pressure loss in the flow development regime. Therefore, we assume $\delta p_{f}+\delta p_{e}=0$ here, which may be a first order approximation and thorough investigation is undergoing. Since the flow is creeping and driven in and out by pressure gradients, the pressure loss at the channel inlet may be expected as the half of that given by Roscoe [34], i.e.

$$
\delta p_{i n}=\frac{16}{\pi} \frac{\mu}{d} \bar{u}_{i n}
$$


When Reynolds number increases, the inertial effect becomes important and cannot be ignored. As Happel and Brenner [35] showed, the pressure loss is no longer linearly proportional to mean velocity once the Reynolds number is greater than 1.6. The typical Reynolds number in a microfluidic flow is less than 1.0, the inertial effect on the pressure loss can therefore be ignored in the present study.

Similarly, the pressure at the channel outlet can be determined by

$$
\begin{aligned}
& p_{\text {out }}=P_{0}+\Delta P_{\text {out }}, \\
& \Delta P_{\text {out }}=\frac{16}{\pi} \frac{\mu}{d} \bar{u}_{\text {out }},
\end{aligned}
$$

where $\bar{u}_{\text {out }}$ is the mean velocity at the outlet. Because the flow from the outlet to the far field down stream is driven by the pressure gradient which inevitably causes a pressure loss $\Delta P_{\text {out }}$, the same amount of pressure has to be generated by the electrokinetic pump in order to allow the fluid move out of the channel to the down stream. Eqs [11, 13] could be very close approxiamtions for the pressure loss due to flow contraction and expansion at the channel inlet and outlet.

Equations $[8,9,11-13]$ can serve as pressure boundary conditions for an EOF at the channel inlet and outlet. Because the pressure is coupled with averaged velocities which are not known a priori, iterations are needed to solve the pressure and velocity field. These boundary conditions can avoid huge computational cost of solving whole reserviors but still capture the flow characteristics such as induced pressure gradients. 


\section{AN EOF THROUGH A STRAIGHT CHANNEL}

As shown in figure 1a, the reservoirs are much larger than the microchannel, so that the flow can rapidly reach the steady state. Now we can apply above pressure boundary conditions to solve Eqs [1-7] for an EOF in the straight channel. With these boundary conditions, we can solve the fully-developed flow regime in the channel. Therefore, the momentum Eq. [2] can be simplised as

$x$ direction:

$$
-\frac{\partial p}{\partial x}+\mu \frac{\partial^{2} u}{\partial y^{2}}-\rho_{e} \frac{\partial(\psi+\phi)}{\partial x}=0
$$

$y$ direction:

$$
-\frac{\partial p}{\partial y}-\rho_{e} \frac{\partial(\psi+\phi)}{\partial y}=0
$$

Eqs [14] and [15] require $\frac{\partial}{\partial x}\left[\frac{\partial p}{\partial x}\right]=0$ and $\frac{\partial}{\partial x}\left[\frac{\partial p}{\partial y}\right]=0$, the pressure field can therefore be expressed as

$$
p(x, y)=C_{1} x+C_{2}+f(y)
$$

where $C_{1}$ and $C_{2}$ are constants. The local net charge density $\rho_{e}$ decays rapidly within the eletric double layer. Becaue the electric double layer is assumed here very small in comparison to the characteristic length scale of the channel, $d$, the gradient of pressure in the $y$ direction can be neglected outside the electric double layer. Because $\partial \psi / \partial x$ is zero due to uniform distribution of zeta potential, substituting Eq. [16] into Eq. [14] leads to

$$
-C_{1}+\mu \frac{\partial^{2} u}{\partial y^{2}}-\rho_{e} \frac{\partial \phi}{\partial x}=0
$$


If there is no applied pressure at the reserviors, most common assumption is that $C_{1}$ is zero. However, the irreversible viscous pressure losses occur at the channel inlet and outlet because of sudden flow contraction and expansion. The schematic diagram of pressure distribution along the central line of the channel from the far flow field of reserviors can be seen in figure 1b. The electrokinetic forces act the same role as a conventional pump where this "electrokinetic pump" compensates not only the frictional kinetic energy loss along the channel but also the pressure losses at the channel inlet and outlet. Because of the existence of a pressure difference between the channel inlet and outlet, the axial velocity profile at the steady and fully-developed regime becomes

$$
u=\frac{\partial \phi}{\partial x} \frac{\varepsilon \zeta_{0}}{\mu}\left\{1-\frac{\cosh (-K y / d)}{\cosh (-K / 2)}\right\}-\frac{C_{1} d^{2}}{8 \mu}\left\{1-4(y / d)^{2}\right\}
$$

where $\zeta_{0}$ is the zeta potential at the surface and $K=k d$.

If the pressure gradient, $C_{1}$, can be determined, the axial velocity profile can be readily expressed by Eq. [18]. This pressure gradient depends on pressure loss at both channel inlet and outlet due to flow contraction and expansion. For the flow in a straight channel considered here, $\bar{u}_{\text {in }}=\bar{u}_{\text {out }}=\bar{u}$. Note, this averaged velocity includes not only electoosmotic velocity but also induced pressure-driven velocity. Combining Eqs [8, 9, 11-13], the amount of pressure generated by the "electrokinetic pump" is

$$
\delta p \approx \frac{32}{\pi} \frac{\mu}{d} \bar{u}
$$

If the channel is long enough that the entry flow developing length is considerably small, the pressure gradient along the pipe, i.e. $C_{1}$, can be estimated by

$$
C_{1}=\frac{\delta p}{L} \approx \frac{32}{\pi} \frac{\mu}{d L} \bar{u}
$$


From Eq. [18], it is readily shown that $C_{1}$ can be neglected, only if

$$
\frac{\partial \phi}{\partial x} \varepsilon \zeta_{0}>>\frac{C_{1}}{8} d^{2}
$$

This equation is actually comparing the magnitude of the electroosmotic velocity and the maximum velocity due to the induced pressure gradient. At the condition that $C_{1}$ is zero, the mean velocity $\bar{u}$ in a straight channel is

$$
\bar{u}=u_{0}\left\{1-\frac{2 \sinh (K / 2)}{K \cosh (K / 2)}\right\} \text {. }
$$

where $u_{0}=\frac{\partial \phi}{\partial x} \frac{\varepsilon \zeta_{0}}{\mu}$. Because the electric double layer considered here is very small compared to channel height, $d, \bar{u}$ is very close to $u_{0}$. Substituting Eq. [20] into Eq. [21], a criterion is obtained to judge whether the induced pressure gradient can be neglected in the straight channel flow:

$$
C_{r}=\frac{4}{\pi} \frac{d}{L}<<1
$$

Eq. [23] clearly shows whether the pressure gradient can be neglected mainly depends on the aspect ratio of the channel length and width $(L / d)$.

For a creeping flow, the pressure loss is a linear function of velocity as shown in Eq. [19], therefore, we can integrate Eq. [18] to get the averaged velocity. With the aid of Eq. [20], we can determine the pressure gradient as

$$
C_{1}=\frac{96 \varepsilon \zeta_{0}}{3 \pi L+8 d} \frac{\partial \phi}{\partial x}\left[1-\frac{2 \sinh (K / 2)}{K \cosh (K / 2)}\right]
$$

Therefore, the velocity profile in the fully-developed flow regime can now readily be solved by Eqs. $[18,24]$. The influence of $L / d$ on the velocity profile can be seen in figures 3 . It is shown that increasing the aspect ratio of $L / d$ is the most efficient way to minimise the flow 
dispersion. We may recommend that the ratio of $L / d$ should not be less than 100 in order to have an ideal plug-like velocity profile. Short $L / d$ has a so-called end effect to the velocity profiles, which has been experimentally confirmed [36]. Simply setting the pressure gradient to zero could lead to significant inaccuracy in numerical simulation results, especially for the channel with a small value of $L / d$.

The induced pressure will decrease the flow rate. The volume flow rate without induced pressure is

$$
Q_{0}=\int_{-d / 2}^{d / 2} \frac{\partial \phi}{\partial x} \frac{\varepsilon \zeta_{0}}{\mu}\left\{1-\frac{\cosh (K y / d)}{\cosh (K / 2)}\right\} d y=d \frac{\partial \phi}{\partial x} \frac{\varepsilon \zeta_{0}}{\mu}\left\{1-\frac{2 \sinh (K / 2)}{K \cosh (K / 2)}\right\}
$$

The volume flow rate reduction caused by the induced pressure is

$$
\delta Q=\frac{C_{1} d^{3}}{12 \mu}
$$

Because the velocity due to induced pressure is very small compared to the electroosmotic velocity, the reduction rate can be estimated as

$$
\frac{\delta Q}{Q_{0}}=\frac{2}{3} C_{r} /\left\{1-\frac{2 \sinh (K / 2)}{K \cosh (K / 2)}\right\}
$$

If the Debye length is negligibly small compared to $d$, then $\delta Q / Q_{0} \approx 2 / 3 C_{r}$. Therefore $C_{r}$ can also be used to estimate the reduction of the volume flow rate due to the induced pressure. The effect of $L / d$ on reduction of volume flow rate is shown in figure 4. It is shown that increasing $L / d$ will reduce the flow reduction rate.

\section{LEAKAGE FLOW IN A T-CHANNEL}

Figure $1 \mathrm{~b}$ shows, for an EOF, the pressure at the channel inlet is less than the reference pressure, $P_{0}$ (which is the value at the far field of the reservoirs ), while at the outlet it is 
greater than $P_{0}$. Therefore, if this straight channel is connected by a side channel which has no imposed pressure (i.e. the pressure at the far field of the reservoir is also $P_{0}$ ) and electric-field free, the contagious flow will occur between the side channel and the main channel due to the induced pressure difference. The leakage flow will be expected from the side channel to the main channel if the intersection is closer to the inlet of main channel while it will be directed from the main channel to the side channel if the intersection is closer to the outlet of the main channel. Harrison et al. [2] and Fan and Harrison [15] reported that the leakage from the side channel to the main channel depends on the location of the intersection. They found noticeable amount of leakage when the intersection was close to the inlet of the main channel. No leakage from the side channel was detected when the intersection was close to the outlet of the main channel. In the latter case, we believe they would have detected the leakage actually from the main channel to the side channel. Because leakage flows are driven by pressure gradients, numerically analysis of the pressure distribution along the $x$ and $y$ axises of T-channels is given below.

Here, the leakage flow between the main and side channels of a T-shaped channel layout, as shown in figure 5, will be tackled in general. The location of the intersection is $L_{1}=L_{2}, L_{1}=2$ $L_{2}$, and $L_{1}=0.5 L_{2}$ respectively. The electric field potentials at the reserviors 1 and 2 are 100 and $0 \mathrm{~V}$. The side channels are electric field free. The uniform zeta potential is applied at the channel walls which is assumed $-0.1 \mathrm{~V}$.

For an EOF in a straight channel, the pressure gradient in the most of the flow field is constant and can be determined, which leads to the analytical solutions of the velocity profile. For the flow in a T-channel, there is no analytical solution, numerical simulation is therefore adopted here. The numerical simulations are generally based on Eqs [1-7] with a set of boundary 
conditions, which are essential components of the model. Non-slip boundary conditions for the velocity are usually applied to the wall. Insulation boundary conditions at the reservoirs for potential $\psi$ and at the channel walls for external electric field $\phi$ are also applied [20].

As discussed in the section 3 , the pressure at the channel inlet and outlet is related to the averaged velocity, the channel thickness and the fluid viscosity. These pressure boundary conditions require the ratio of $L / d$ to be sufficiently large, so that fully-developed boundary conditions can be applied at the end of the flow development regime. From Eqs [8-13], the boundary conditions for T-channel can therefore be obtained as

Channel inlet at the reservoir 1: $\quad \frac{\partial u}{\partial x}=0, \quad v=0, \quad p=P_{0}-\frac{16}{\pi} \frac{\mu}{d} \bar{u}_{i n}$,

Channel outlet at the reservoir 2: $\frac{\partial u}{\partial x}=0, \quad v=0, \quad p=P_{0}+\frac{16}{\pi} \frac{\mu}{d} \bar{u}_{\text {out }}$,

Channel inlet or outlet at the reservoir 3: $\frac{\partial v}{\partial y}=0, \quad u=0, \quad p=P_{0} \pm \frac{16}{\pi} \frac{\mu}{d} \bar{v}_{s}$,

where $\bar{u}_{\text {in }}, \bar{u}_{\text {out }}$ and $\bar{v}_{s}$ are the mean velocities at the channel inlet and outlet, and side channel respectively. If the leakage flow is from the reservoir 3 to the main channel, then pressure at the inlet is smaller than reference pressure $P_{0}$ (negative sign in Eq. [30]), otherwise the pressure at the side channel outlet is larger than $P_{0}$ (positive sign in Eq. [30]). These boundary conditions reflect the physical mechanisms governing the flow and are needed in theoretical and numerical solution to the flow field. Since the velocity field needs to be determined and the pressure at the channel inlets and outlets are coupled with the averaged velocities, interations are needed to solve the flow field. Here, the flow and electric solvers of CFD-ACE [31] are used to solve the flow and electric fields. 
The simulation results in the straight channel have shown that the induced pressure may have negligible influence on the flow if $L / d$ is large. However, the induced pressure will still cause unexpected experimental phenomenon. For example, Fan and Harrison [15] observed a leakage flow from the side channels, despite how small it is, which could be due to the induced pressure.

In figures $6-8$, comparisons of the pressure distribution along the $x$-axis and the $y$-axis between the models using the pressure boundary conditions as Patankar and $\mathrm{Hu}$ [20] where they assumed atmospheric pressure at the channel inlets and outlets and the present pressure boundary conditions as given by Eqs [28-30]. For the sake of clarity, we denote the model with boundary conditions of Patankar and $\mathrm{Hu}[20]$ as model 1 and the present model with pressure boundary conditions of Eqs [28-30] as model 2. In these simulations, Reynolds number is about 0.1 , therefore the inertia effect is insignificant.

In figure 6 , the intersection is in the middle of the main channel, i.e. $L_{1}=L_{2}$. The induced pressure gradients both in the $x$-axis and the $y$-axis predicted by the model 2 are larger than the model 1 . Because the pressure gradient in the side channel in figure $6 \mathrm{~b}$ is very small, the leakage is negligible for both models. Since the flow in the intersection area is also partially driven by the pressure gradient, we can see from figure 6 a that the pressure is built up before the fluid enters the intersection area and then it drops to allow the fluid to move into the channel again. The mechanism of the pressure change due to sudden flow expansion and contraction at the intersection area is the same as that at the channel inlets and outlets. The result shown in figure 6a is another evidence that the pressure at the inlets and outlets should be different to the reference pressure, $P_{0}$. The pressure gradients in the intersection in the $x$ 
direction for both models are nearly the same because of nearly identical flow rates. This is further confirmed in the simulation results presented in figures 7 and 8.

The difference between figures 7 and 8 is the location of intersection. $L_{1}$ is $500 \mu \mathrm{m}$ and $L_{2}$ is $1000 \mu \mathrm{m}$ in figure 7 while they are 1000 and $500 \mu \mathrm{m}$ respectively in figure 8 . The different location of intersection leads to different leakage flow direction. Because of larger induced pressure gradients, pronounced leakages from the side channel to the main channel and vice versa are predicted by the model 2 in figures $7 \mathrm{~b}$ and $8 \mathrm{~b}$. Fan and Harrison [15] observed a leakage only from the side channel to the main channel where the intersection is closer to the entrance of the main channel. They reverse the EOF direction so that the intersection is closer to the outlet but they did not detect the leakage. From the result presented in figure 8 , we expect a leakage flow from the main channel to the side channel to occur. The model 1 significantly underpredicts or fails to predict the leakage.

From figure $6 \mathrm{~b}$, we can see the pressure at the side channel inlet is nearly the same as the reference pressure, $P_{0}$, due to neligibly small amount of leakage (the intersection is in the middle of main channel). More pronounced pressure difference to $P_{0}$ is found at the inlet of the side channel in both figures $7 \mathrm{~b}$ and $8 \mathrm{~b}$, which is caused by larger amount of leakages. If we compare the magnitude of the pressure drops at the inlet or the outlet with those at the intersection area, where the pressure drops are caused by the same mechanism, the pressure drops at the channel inlet and outlet are larger which are shown in figures $6 \mathrm{a}, 7 \mathrm{a}$ and $8 \mathrm{a}$. This may attribute to the fact that the one side of the intersection area is still the main channel wall. Therefore, the flow is not fully expanded or contracted in the intersection area. At the same time, the zeta potential at this wall still generates pressure which offsets some pressure loss. 
Moreover, the length of the intersection is too short to allow the outflow to be fully-developed, which may lead to a smaller pressure loss.

If the contagious leakage from a side channel needs to be prevented, the position of the intersection should be designed to be closer to the outlet of the main channel. Alternatively, it can be controlled by changing pressure at the side channel reservior, or applying an electric potential there [10]. In the present work, a two-dimensional calculation is carried out in order to clearly identify the physical mechanism of a leakage flow. The precise prediction of leakage will depend on actual geometry of channels.

\section{CONCLUSIONS}

The induced pressure in EOFs through channels does exist, which may have significant effect on enhancing flow dispersion rate. The reduction of volume flow rate due to this induced pressure can only be ignored for channels with large aspect ratio, $L / d$. Moreover, the pressure losses at the channel inlet and outlet, where the flow contracts and expands, are the key to understanding the leakage flow. The new boundary conditions at the channel inlet and outlet have been established which are capable of capturing the mechanism of the induced pressure gradient. The pressure distribution in a T-shaped channel shows that the leakage flow can occur from the main channel to the side channel or vice versa, depending on the location of the intersection.

\section{ACKNOWLEDGEMENT}

This work was carried out as part of the $\mu$ FAST project with support from the Medical Research Council of UK under grant reference 57719. 


\section{References}

1. Harrison, D. J., Manz, A., Fan, Z., Lüdi, H. and Widmer, H. M., Anal Chem. 64, 1926 (1992).

2. Harrison, D. J., Fluri, K., Seiler, K., Fan, Z., Effenhauser, C. S. and Manz, A., Science 261, 895 (1993).

3. Hadd, A. G., Raymond, D. E., Halliwell, J. W., Jacobson, S. C. and Ramsey, J. M., Anal. Chem. 69, 3407 (1997).

4. Herr, A. E., Molho, J. I., Santiago, J. G., Mungal, M. G. and Kenny, Y. W., Anal. Chem. 72, 1053 (2000).

5. Tang, T., Badal, M. Y., Ocvirk, G., Lee, W.E., Bader, D.E., Bekkaoui, F. and Harrison, D. J., Anal. Chem. 74, 725 (2000).

6. Yan, C., Dadoo, R., Zhao, H., Zare, R. N. and Rakestraw, D. J., Anal. Chem. 67, 2026 (1995).

7. Dittmann, M. M. and Rosing, G. P., J. Chromatogr., A. 744, 63 (1996).

8. Choudhary, G. and Horváth, C., J. Chromatogr., A. 781, 161 (1997).

9. Xiang, R. and Horváth, C., Anal. Chem. 74, 762 (2002).

10. Seiler, K., Fan, Z. H., Fluri, K. and Harrison, D. J., Anal Chem. 66, 3485 (1994).

11. McCormick, R. M., Nelson, R. J., Alonso-Amigo, M.G., Benvegnu, D. J. and Hooper, H. H., Anal Chem. 69, 2626 (1997). 
12. Waters, L.C., Jacobson, S.C., Kroutchinina, N., Khandurina, J., Foote, R. S. and Ramsey, J. M., Anal. Chem. 70, 158 (1998).

13. Tallarek, U., Rapp, E., Scheenen, T., Bayer, E. and Van As, H., Anal. Chem. 72, 2292 (2000).

14. Polson, N. A., Hayes, M. A., Anal Chem. 312A-319A. (2001).

15. Fan, Z H. and Harrison, D. J., Anal Chem. 66, 177 (1994).

16. Seiler, K., Harrison, D. J. and Manz, A., Anal Chem. 65, 1481 (1993).

17. Soderman, O. and Jonnson, B., J. Chem. Phys. 105, 10300 (1996).

18. Mala, M., Li, D., Werner, C., Jacobasch, H. and Ning, Y. Int. J. Heat Fluid Flow 18, 489 (1997).

19. Molho, J. I., Herr, A. E., Kenny, T. W., Mungal, M. G., Deshpande, M., Gilbert, J. R., Garguilo, M. G., Paul, P. H., St. John, P. M., Woudenberg, T. M. and Connell, C., MEMS 66, 69 (1998).

20. Patankar, N.A. and Hu, H.H., Anal Chem. 70, 1870 (1998).

21. Ermakov, S. V., Jacobson, S. C. and Ramsey, J. M., Anal. Chem. 70, 4494 (1998).

22. Hu, L., Harrison, J. D. and Masliyah, J. H., J. Colloid Interface Sci. 215, 300 (1999).

23. Mitchell, M. J., Qiao, R. and Aluru, N. R. J. MEMS 9, 435 (2000).

24. Bianchi, F., Ferrigno, R. and Girault, H. H., Anal. Chem. 72, 1987 (2000).

25. Yang, R. J., Fu, L. M. and Lin, Y. C., J. Colloid Interface Sci. 239, 98 (2001). 
26. Santiago, J. G., Anal. Chem. 73, 2353 (2001).

27. Dutta, P. and Beskok, A., Anal. Chem. 73, 1979 (2001).

28. Liapis, A. I. and Grimes, B. A., J. Chromatography A 877, 181 (2000).

29. Sadri, R. M. and Floryan, J.M., Computers \& Fluids 31, 133 (2002).

30. Hunter, R. J., "Foundations of Colloid Science ( $2^{\text {nd }}$ ed.)." Oxford University Press, Oxford, 2001 .

31. CFD Research Corporation, Cummings Research Park, 215 Wynn Drive, Huntsville, AL 35805, USA.

32 Ferziger, J.H. and Perić, M., "Computational methods for fluid dynamics ( $3^{\text {rd }}$ ed.)" Springer, Berlin, 2002.

33. Chen R.Y., J. Fluid Eng. 95, 153 (1973).

34. Roscoe, R., Philosophical Magazine 40, 338 (1949).

35. Happel, J. and Brenner, H., "Low Reynolds Number Hydrodynamics." Prentice-Hall, Inc., Englewood Clifs, N.J., 1965.

36. Ross, D., Johnson, T. J. and Locascio, L.E., Anal Chem. 73, 2509 (2001). 


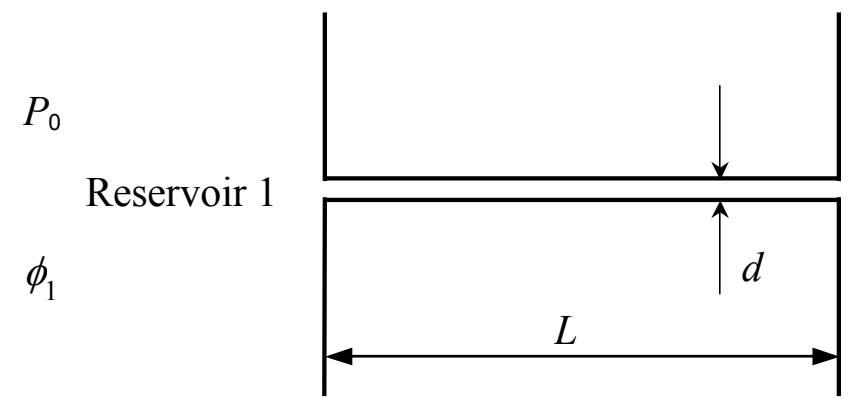

(a)

$$
P_{0}
$$

Reservoir 2

$$
\phi_{2}
$$

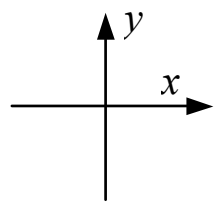

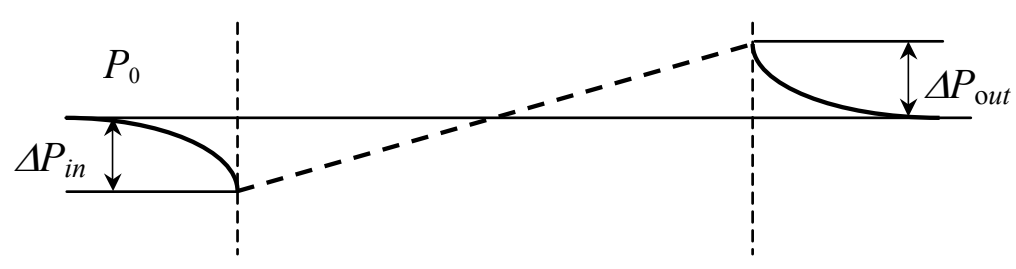

(b)

Figure 1. Schematic diagram of a) a two-dimensional microchannel; b) pressure dissitribution along the $x$-axis of the channel. $P_{0}$ is the reference pressure at the far field of the reserviors. The electroosmotic flow is from the reservoir 1 to the reservoir 2. 


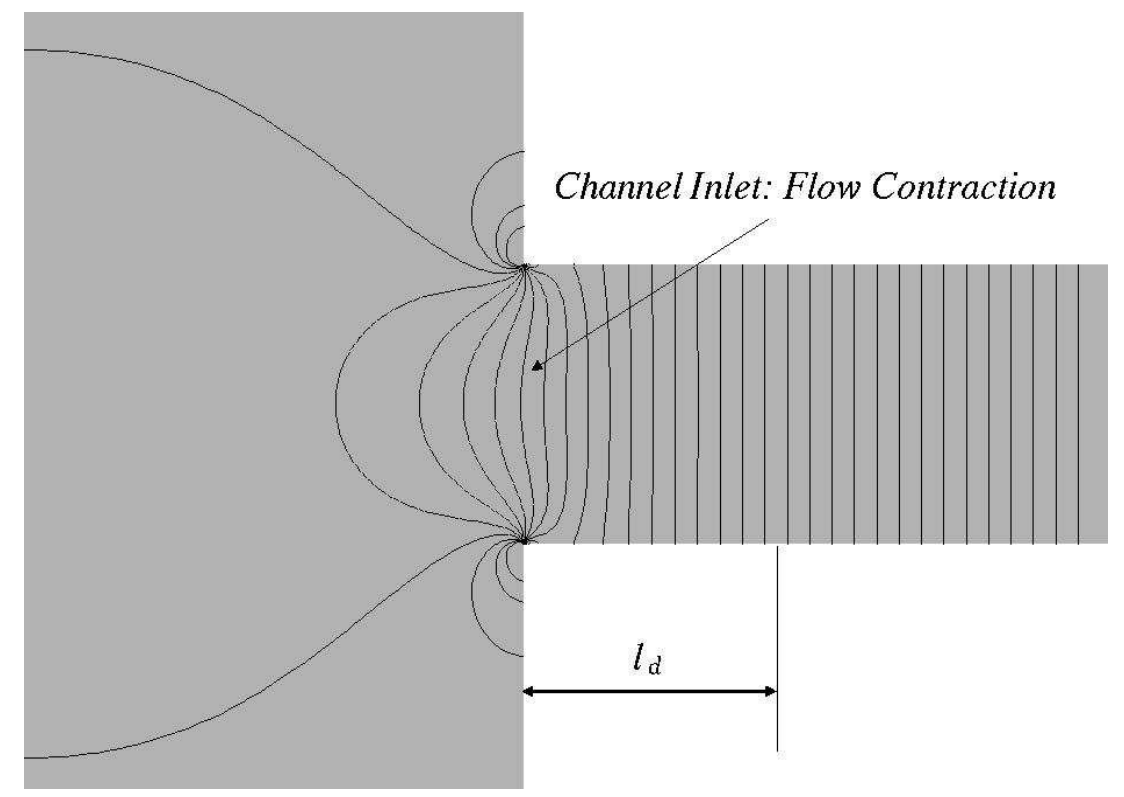

(a)

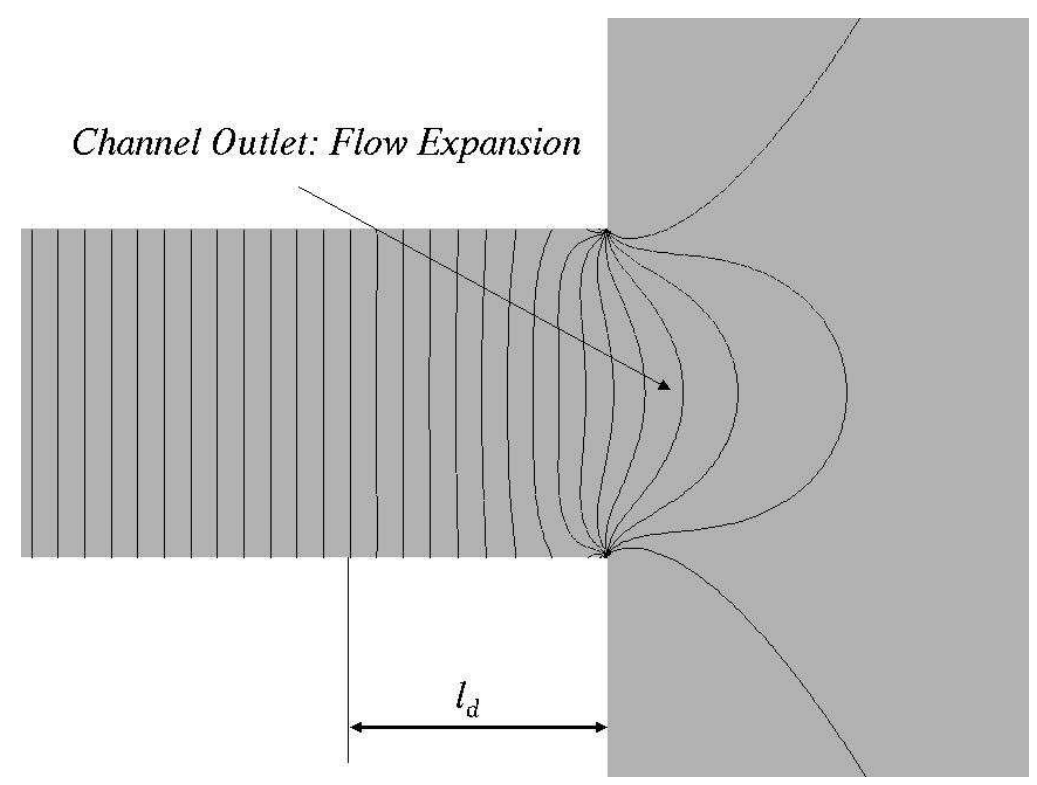

(b)

Figure 2. Pressure contours and flow development length $l_{d}$ at a) the channel inlet and b) the channel outlet. Only small part of the reservoirs connected to the channel is shown. 


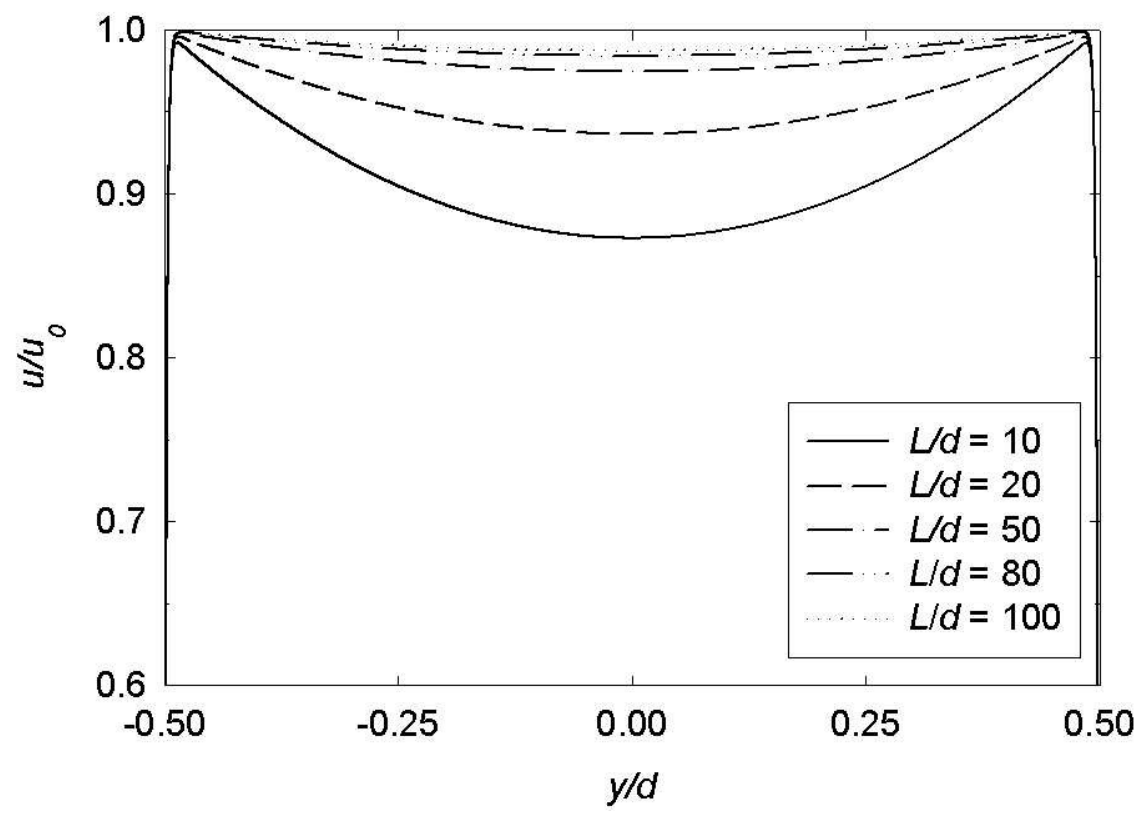

Figure 3. The effect of the aspect ratio of length and width, i.e. $L / d$, on the axial velocity profile, The ratio of channel thickness, $d$, to the Debye length, $k^{-1}, d k=500$.

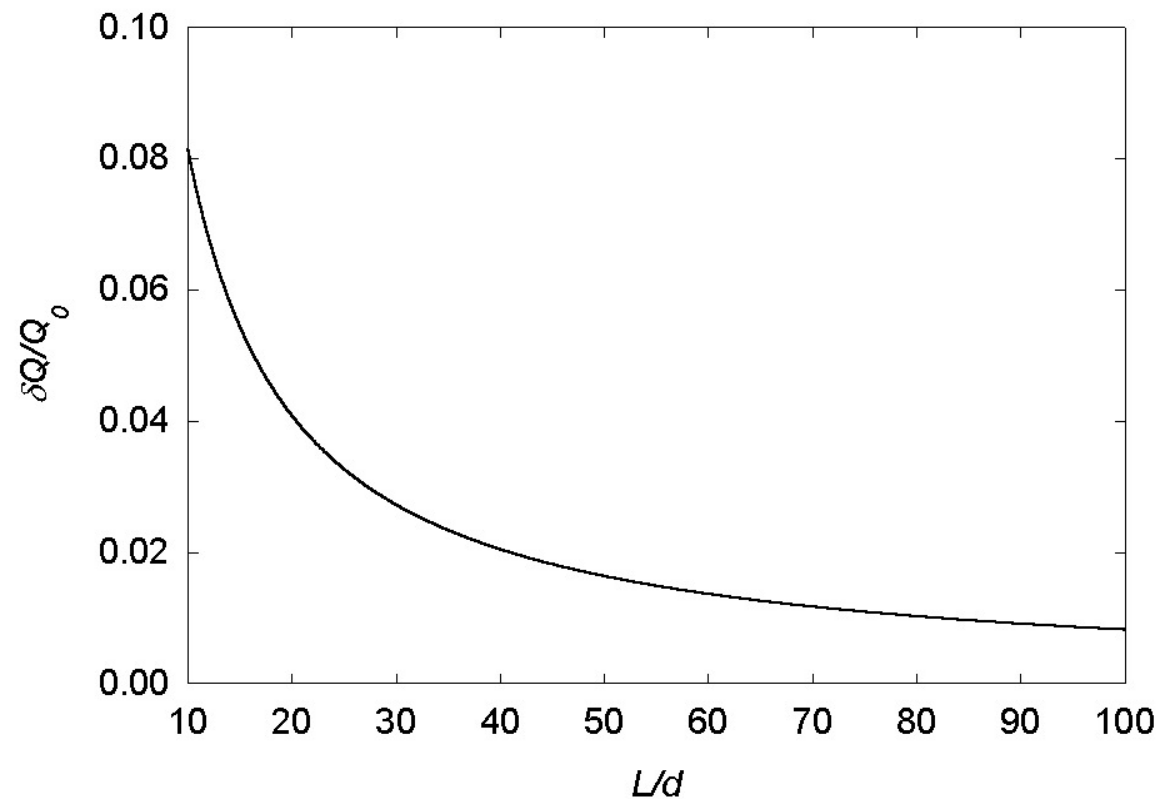

Figure 4. The effect of $L / d$ on reduction of volume flow rate due to the induced pressure gradient, The ratio of channel thickness, $d$, to the Debye length, $k^{-1}, d k=500$. 


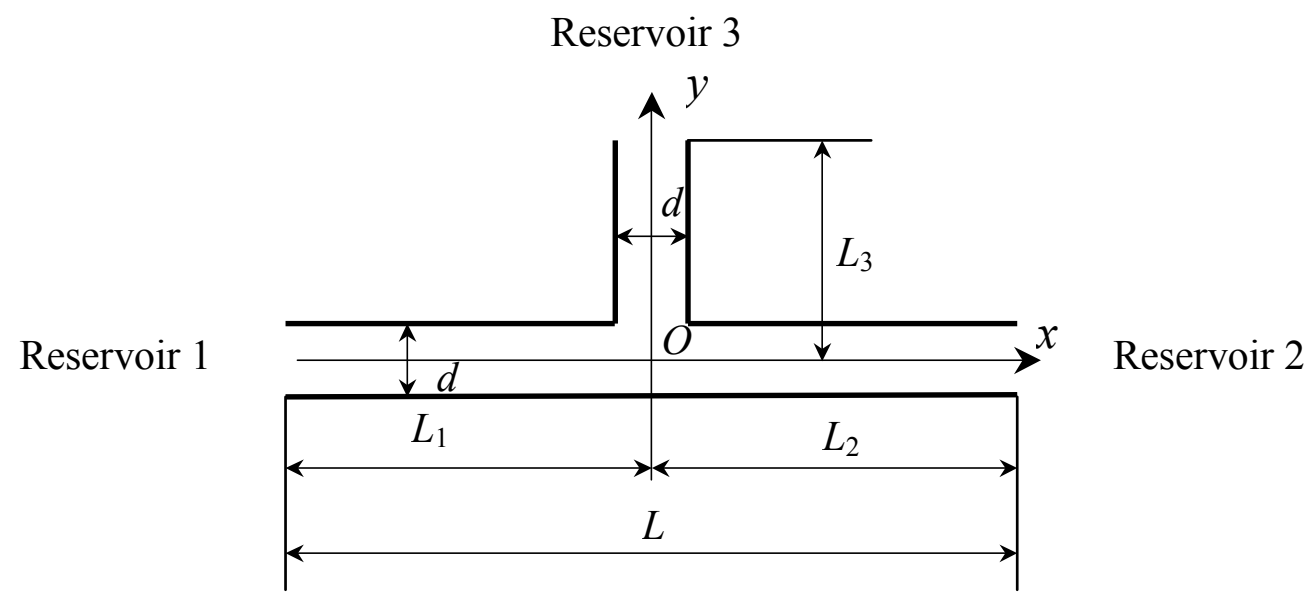

Figure 5. Schematic of the T-shaped channel, $d=30 \mu \mathrm{m}, L=1500 \mu \mathrm{m}$ and $L_{3}=500 \mu \mathrm{m}$. 

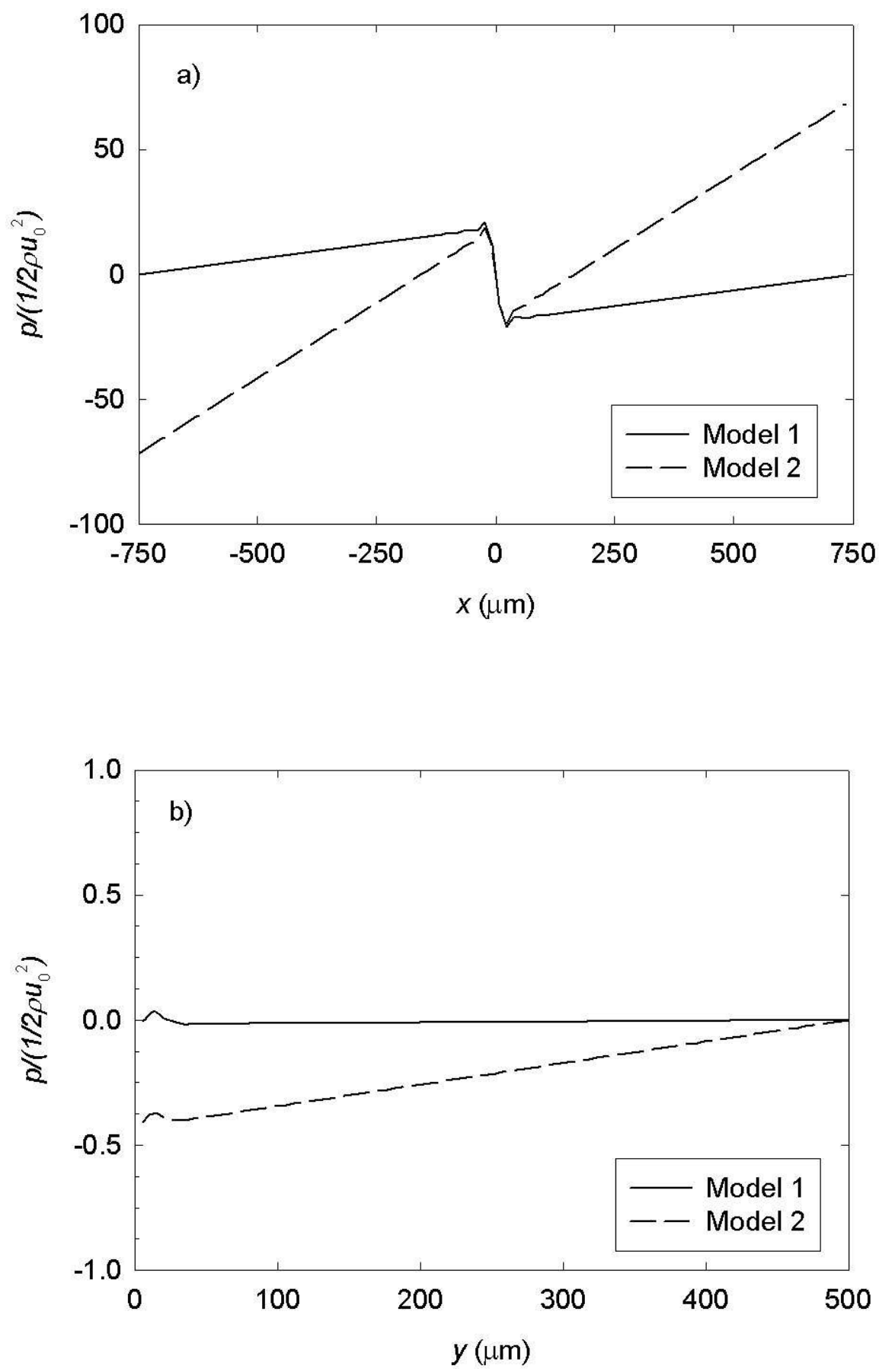

Figure 6. Pressure distribution along a) $x$-axis b) $y$-axis. Model 1 uses the reference pressure, $P_{0}$, for the pressure at the channel inlets and outlets, and Model 2 uses Eqs [28-30] for boundary conditions. The intersection is in the middle of main channel. 

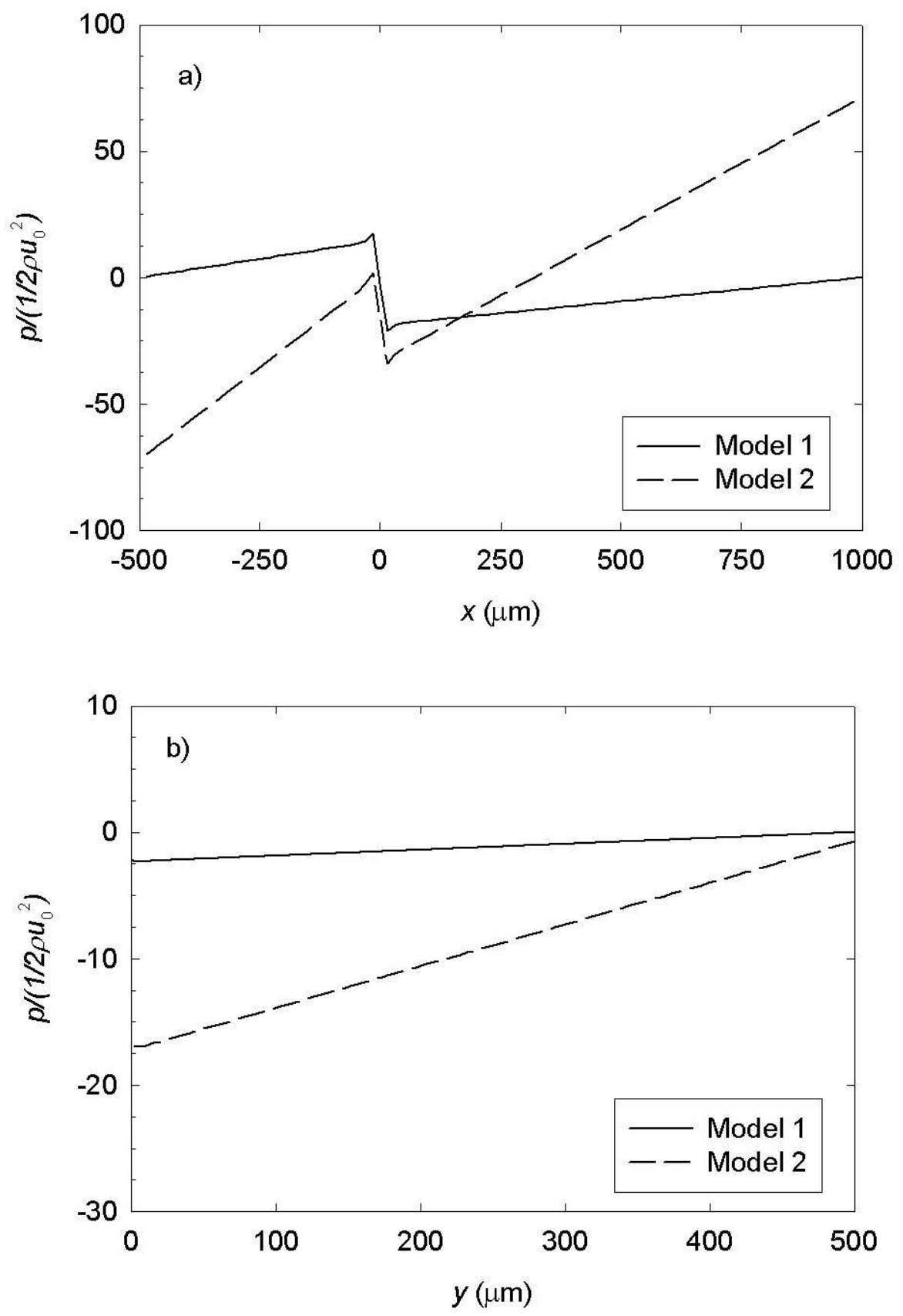

Figure 7. Pressure distribution along a) $x$-axis b) $y$-axis. Model 1 uses the reference pressure, $P_{0}$, for the pressure at the channel inlets and outlets, and Model 2 uses Eqs [28-30] for boundary conditions. The intersection is close to the main channel inlet. 

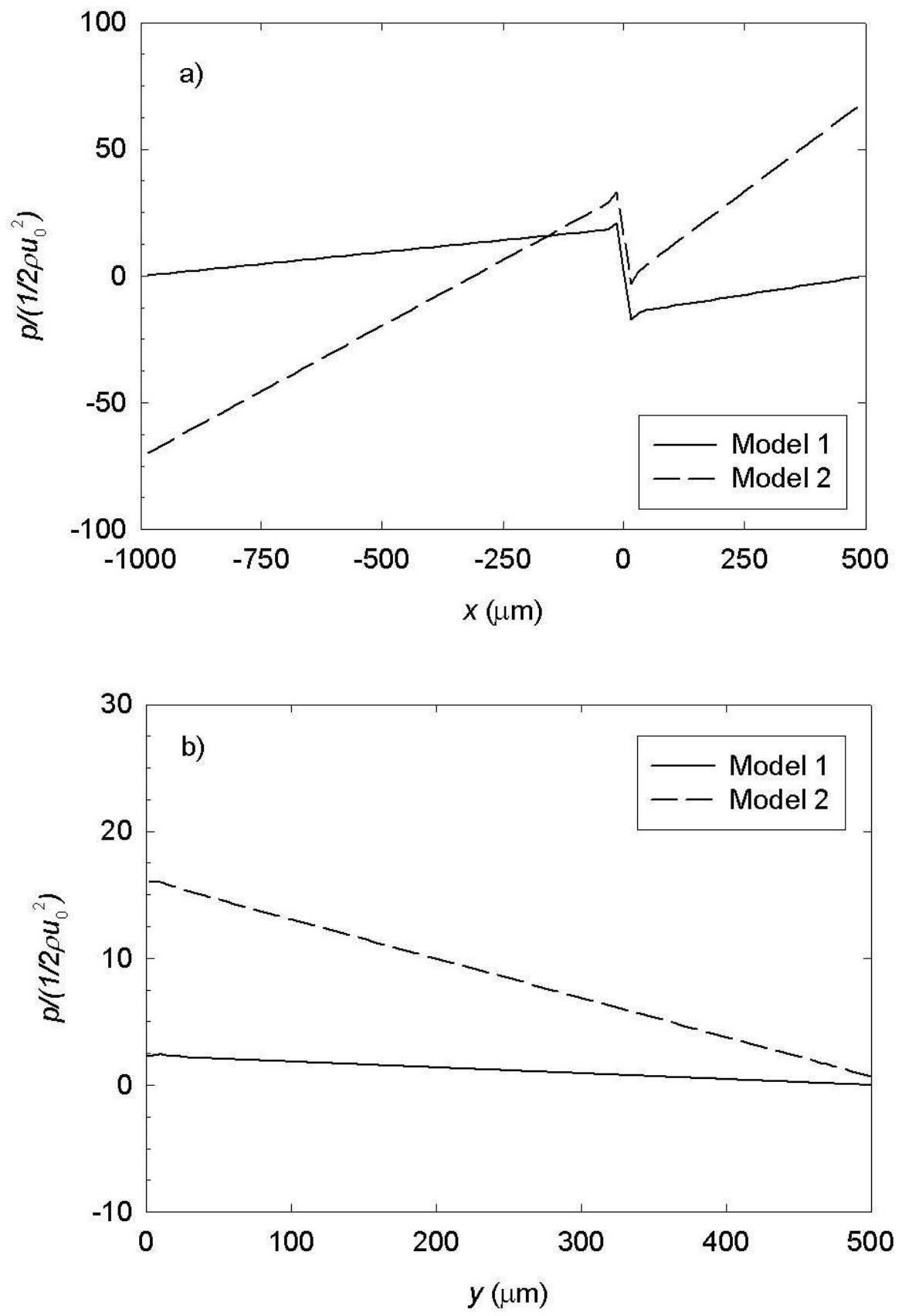

Figure 8. Pressure distribution along a) $x$-axis b) $y$-axis. Model 1 uses the reference pressure, $P_{0}$, for the pressure at the channel inlets and outlets, and Model 2 uses Eqs [28-30] for boundary conditions. The intersection is close to the main channel outlet. 